The Astrophystcax JournaL, 228:L55-L58, 1979 March 1

(C) 1979. The American Astronomical Society. All rights reserved. Printed in U.S.A.

\title{
RELATIONS BETWEEN THE EMISSION SPECTRA AND RADIO STRUCTURES OF QUASARS*
}

\author{
George K. Miley \\ Sterrewacht, Huygens Laboratorium, Leiden, The Netherlands; and Lick Observatory, University of California, Santa Cruz \\ AND
}

Joseph S. MilLER

Lick Observatory, Board of Studies in Astronomy and Astrophysics, University of California, Santa Cruz, CA 96054 Received 1978 October 6; accepted 1978 December 1

\begin{abstract}
We present evidence that the emission spectra and radio structures of low-redshift quasars are related statistically. Our sample comprises 34 quasars with $z<0.70$ and known radio structures. Objects with the broadest and most irregular emission lines are associated with extended radio sources, while those with relatively narrow emission lines with smooth profiles tend to have compact radio structures. There is also a significant tendency for Fe II emission lines to be found preferentially in quasars with compact radio structure.
\end{abstract}

Subject headings: quasars - radio sources: general

\section{INTRODUCTION}

Until now few connections have been found between the optical and radio properties of quasars. In this Letter we shall present evidence for the existence of some relationships between the optical emission-line spectra and the radio structures. About half of all quasars found in low-frequency radio surveys (e.g., 3C, $4 \mathrm{C}, \mathrm{PKS}, \mathrm{B} 2)$ have relatively symmetric double-lobed radio structure extending to more than $100 \mathrm{kpc}$ from the QSO. Often called $D_{1}$ quasars (Miley 1971), their radio structures are indistinguishable from those of most high-luminosity radio galaxies (e.g., Cygnus A), except that on the average they have stronger compact nuclear radio cores (Miley and Hartsuijker 1978). A typical example is 3C 47. About one-third of lowfrequency quasars are dominated by their variable, flat-spectrum nuclear components. These are often isolated compact sources (e.g., PKS 1510-08), but sometimes there is also extended structure present. In this case the extended structure is usually smaller than $50 \mathrm{kpc}$ and one-sided, that is, distributed asymmetrically with respect to the optical QSO. Examples of these asymmetric $\left(\mathrm{D}_{2}\right)$ quasars are $3 \mathrm{C} 273,3 \mathrm{C} 345$, and $3 \mathrm{C}$ 454.3. Finally, there are a smaller number $(\sim 10 \%)$ of quasars, like 3C 48 and 3C 147, which have compact radio structures ( $\leq 100 \mathrm{pc}$ ) and radio spectra which do not vary appreciably and show cutoffs at frequencies below $1 \mathrm{GHz}$ (LFC quasars).

A search for extended radio components close to several compact quasars (Miley and Hartsuijker 1978) failed to detect any emission with an upper limit of $1 \%$ of the central compact source, indicating that there are definite qualitative differences between the various structural types. We therefore decided that an attempt to seek connections between the different radio morpholgies and other QSO parameters might prove fruitful.

\footnotetext{
* Lick Observatory Bulletin, No. 817.
}

\section{EMISSION SPECTRA}

Although the emission spectra of low-redshift QSOs differ considerably from object to object, the Balmer series of hydrogen is always present, and helium lines are also often seen. These permitted $\mathrm{H}$ and $\mathrm{He}$ lines usually have widths corresponding to velocities of several thousand kilometers per second. Provided the redshift is large enough, $\mathrm{Mg}$ II $\lambda 2800$ is also generally visible and usually has a width similar to that of the Balmer lines. The broad permitted lines contrast with the narrow forbidden lines of $\mathrm{O}$ III, O II, S II, Ne III, and $\mathrm{Ne} \mathrm{v}$ which are commonly seen in low-redshift QSO spectra.

In addition to the usual forbidden and permitted emission lines discussed above, the spectra of a few low-redshift QSOs show blended lines of several permitted multiplets of Fe II between $4450-4650 \AA$ and 5100-5600 $\AA$ (Phillips 1978). These features are very common in Seyfert 1 galaxies, but are considerably rarer in QSOs.

We have tried to accumulate all spectra of lowredshift quasars having known radio structures which have been taken at Lick Observatory over the last few years. The spectra were obtained with the image-tube scanner (ITS) at the Cassegrain focus of the $3 \mathrm{~m}$ Shane telescope (Robinson and Wampler 1972; Miller, Robinson, and Wampler 1976). The resolution of the spectra is about $8 \AA$.

We have restricted our sample to low-redshift quasars $(z<0.7)$ since in general their radio structures are better determined. Our cutoff $z=0.70$ was chosen so that $\mathrm{H} \beta$ could be observed with the ITS. The resulting sample of 34 quasars, though not complete or uniform, involves no known bias with respect to their radio properties. Column (1) of Table 1 lists the quasars in the notation of the catalog of Burbidge, Crowne, and Smith (1977), some alternative names by which the objects are known are in column (2), and the redshifts 
are in column (3). The largest angular size (LAS) observed for the radio structure is given in column (4), and the appropriate reference and corresponding linear 19: size are listed in columns (5) and (6). Column (7) indicates whether the source size is smaller than 50 $\rightarrow \rightarrow \mathrm{kpc}(\mathrm{C})$, or, if more extended, whether it has symmetric (SE) or asymmetric (AE) structures.

One of the potentially most useful and least modeldependent parameters of a broad-line emission spectrum is the line profile. In our sample, the shape of the $\mathrm{H} \beta$ profile varied considerably from object to object. In some cases it was relatively simple, but in others it appeared quite complex; a few objects had a composite $\mathrm{H} \beta$ profile with broad and narrow components superposed. Examples are shown in Figure 1. We have graded the complexity of the $\mathrm{H} \beta$ profile of each object on a scale from 0 (simple) to 3 (very complex), and these values are given in column (8) of Table 1 . It should be stressed that these assessments are rather qualitative and are definitely influenced by the signal-to-noise ratio in the data, so the numbers should be taken as rough indicators only. More than half the profiles were significantly more extended to the red than to the blue. Such an asymmetry has been noted previously for the broad lines in some Seyfert galaxies and QSOs (e.g., Osterbrock 1977). We graded the degree of asymmetry on a scale from 0 (symmetric) to 3 (highly asymmetric) and list the values in column (9). Again, these are approximate, visual estimates. In columns (10) and (11) we list two parameters which characterize the widths of the $\mathrm{H} \beta$ profiles: the rest-frame velocities corresponding to the full width at half-maximum intensity (FWHM) and the half-width at quarter-maximum intensity on the blue side of the line (HWQMb). We restrict the measurement of the HWQMb to the blue side because the red part of the quarter intensity is usually confused by [O III] $\lambda \lambda 4959,5007$. The FWHM and HWQMb are illustrated in Figure 1. Their uncertainties in the table are typically a few hundred $\mathrm{km} \mathrm{s}^{-1}$. When both the $\mathrm{H} \beta$ and $\mathrm{Mg}$ II lines were observed, there was usually

TABLE 1

Quasars in Sample

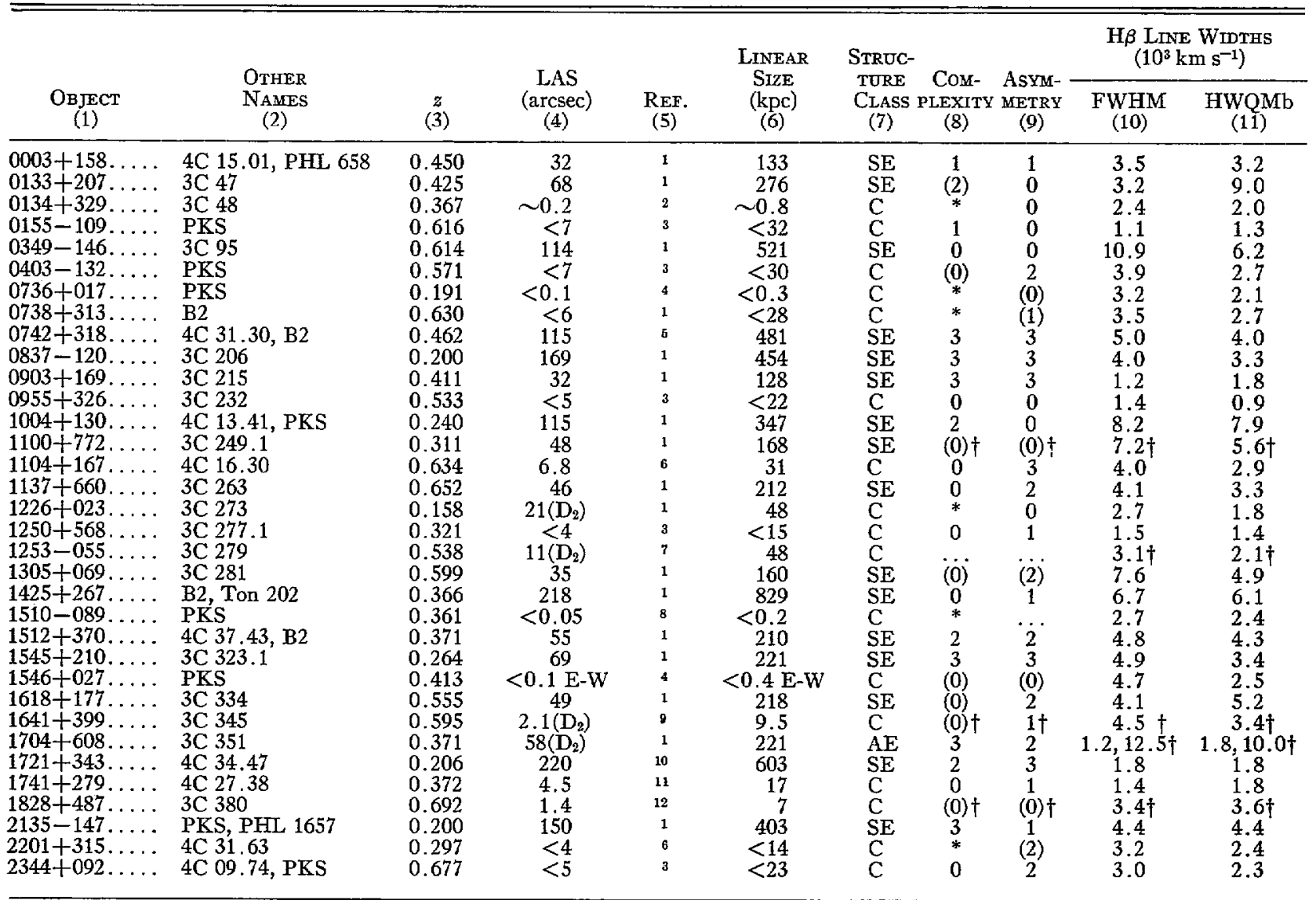

* Spectrum confused by $\mathrm{Fe}$ II emission.

$\dagger$ Parameters measured from $\mathrm{Mg}$ II $\lambda 2800$.

${ }^{1}$ Miley and Hartsuijker 1978. ${ }^{2}$ Palmer et al. $1967 .{ }^{3}$ Miley 1971. ${ }^{4}$ Bentley et al. $1976 .{ }^{4}$ Fanti et al. $1977 .{ }^{6}$ Wardle and Miley 1974. ${ }^{7}$ Lyne 1972. ${ }^{8}$ Donaldson et al. 1969. ${ }^{9}$ Davis et al. 1977. ${ }^{10}$ Conway et al. 1977. ${ }^{11}$ Stannard and Neal 1977. ${ }^{12}$ Wilkenson et al. 1974. 


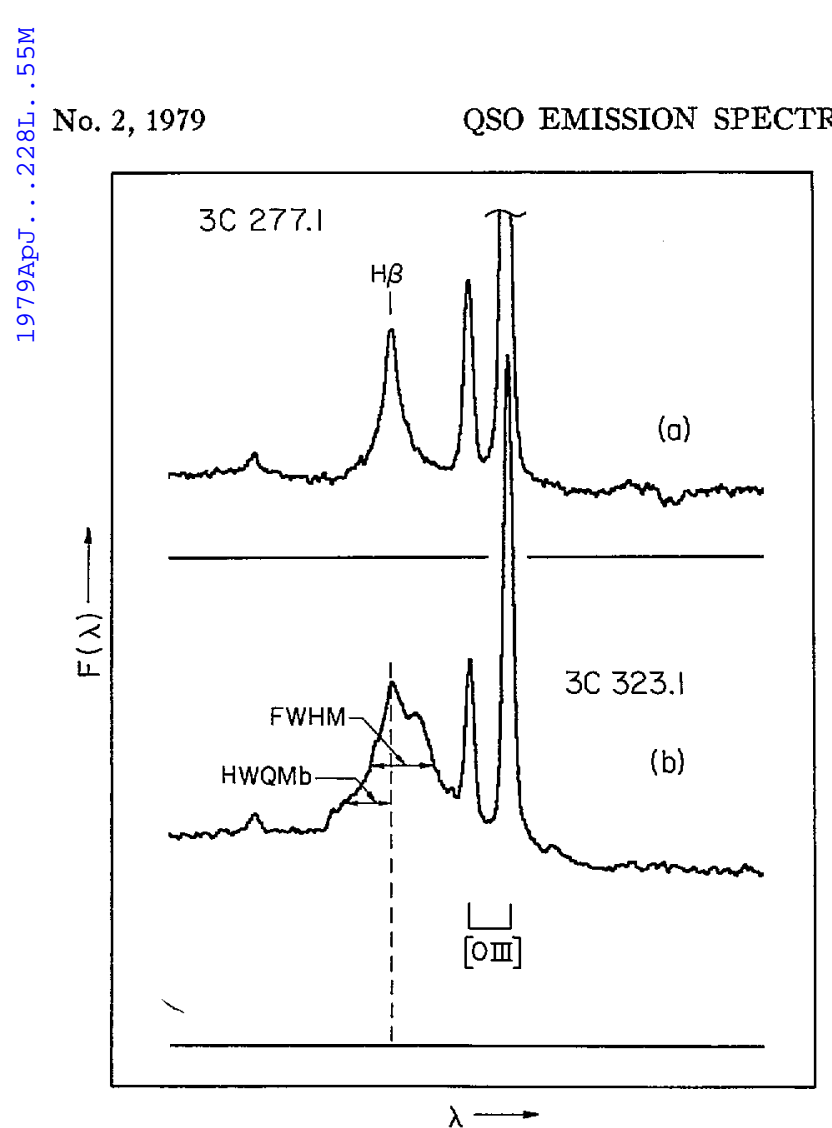

FIG. 1.-The spectral region of $\mathrm{H} \beta$ and $[\mathrm{O} \Pi \mathrm{II}] \lambda \lambda 4959,5007$, in 3C 277.1 and 3C 323.1 in the emission frame. Note how the FWHM and HWQMb are defined. reasonable agreement between the two profiles; the only exception was $3 \mathrm{C} 351$ (see below). In three cases for which no $\mathrm{H} \beta$ data were available, we have therefore listed parameters for the $\mathrm{Mg}$ II lines instead. Values in Table 1 that have particularly large uncertainties are enclosed in parentheses.

\section{DISCUSSION OF THE OBSERVATIONAL DATA}

Table 1 contains 17 extended and 17 compact radio sources. Examination has revealed significant differences in the optical spectra of the two classes of sources. The most striking difference involves the shape and width of the profiles. Figures $2 a$ and $2 b$ show comparison histograms for the FWHM and HWQMb of the two radio classes. All with the broadest lines, the six with the largest FWHM and the 10 with the largest $H W Q M b$, are quasars with symmetric extended radio structures. One of the most powerful methods of testing whether two distribution functions represent the same population is the Kolmogorov-Smirnov test on the cumulative distributions (Knuth 1969). Applying the two-tailed version of this test to the data in Figure 2, we find that there is about a $1 \%$ chance that the two FWHM distributions have been drawn from the same population; for the HWQMb the chance is less than $0.1 \%$. Thus it appears that the width of the wings separates the two groups most clearly, since the HWQMb is more sensitive to these wings than is the FWHM. Note that 3C 351 has been omitted from this comparison. Its $\mathrm{Mg}$ II profile is extremely broad, but

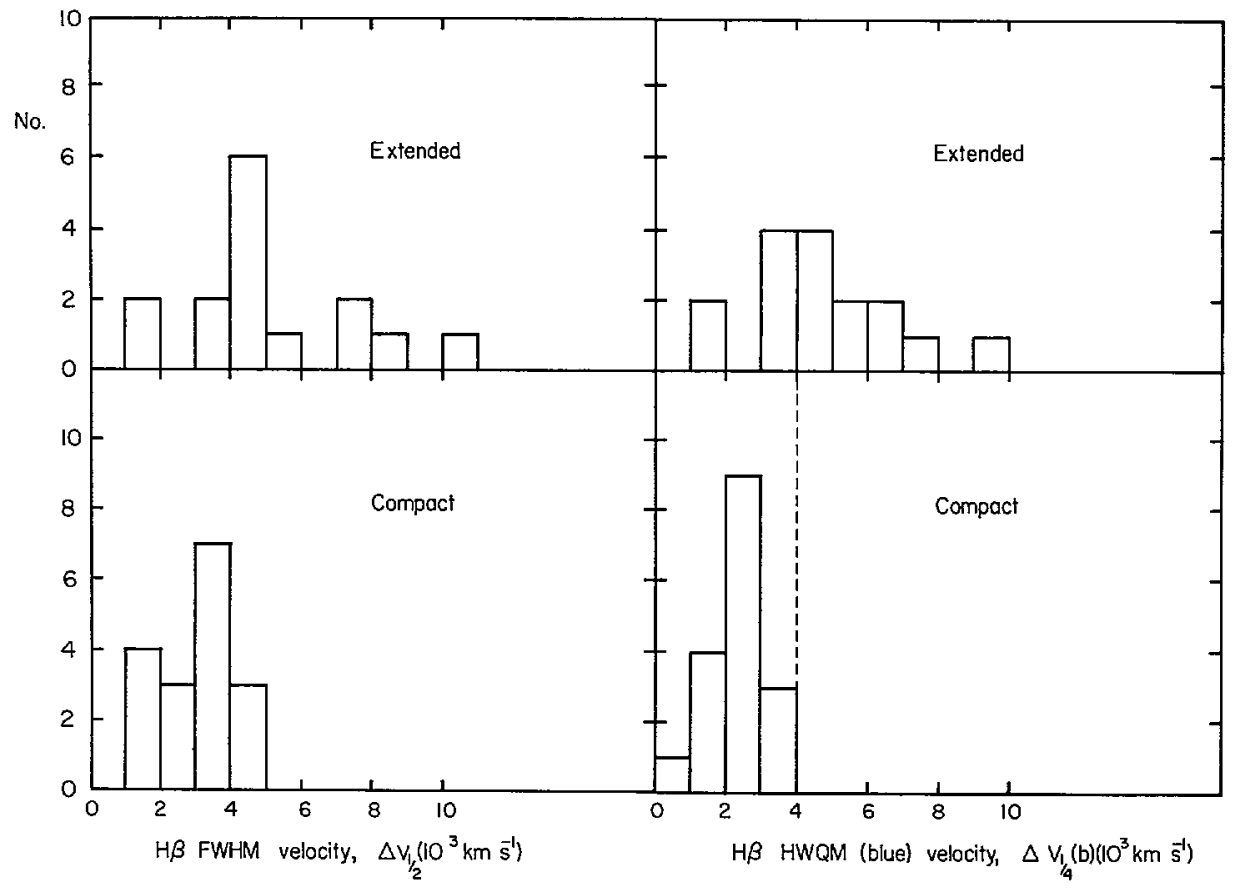

FIG. 2.-Histograms showing the differences in $\mathrm{H} \beta$ line widths for the extended and compact sources. The line widths are velocities in the emitted frame corresponding to the full width at half-maximum intensity (FWHM) and the half-width at quarter-maximum intensity on the blue side (HWQMb). The latter is more sensitive to weak broad components. Note that almost two-thirds of the extended sources, but none of the compact sources, have HWQMb greater than $4000 \mathrm{~km} \mathrm{~s}^{-1}$ (dotted line). 
its $\mathrm{H} \boldsymbol{\beta}$ profile is dominated by a very narrow component surrounded by a weak broad feature. The source $3 \mathrm{C}$ 351 also has a highly anomalous radio structure. At 5 $\mathrm{GHz}$ the flux ratio for the two extended components is more than 20, compared with a typical value of 2 or 3 .

Not only do the line widths differ for compact and extended quasars, but the degree of complexity of the profile is also significantly different on average. All 10 of the complex profiles (complexity definitely $\geq 2$ ) occur in extended quasars. This effect seems to be independent of the correlation involving the line widths, since extended quasars with the narrowest lines are as likely to be complex as those with the broadest lines.

We encountered two other suggestive differences between the emission spectra of the compact and extended quasars in Table 1. Five of the objects show strong Fe Ir emission, and one, B2 0738+313, probably has weak Fe II emission. None of these objects has radio emission extending more than $50 \mathrm{kpc}$ from the center. Thus there seems to be a strong tendency for Fe II emission to occur preferentially in quasars having compact radio structures. From the available data, there is about a $3 \%$ chance that this result is an accident. All five strong Fe II quasars (about one-third of our compact sample) and about one-half of all Seyfert 1 galaxies have Fe $\Pi(\lambda 4570+\lambda 5190+\lambda 5320) /$ $\mathrm{H} \beta>0.5$, but no spectrum of a Seyfert 2 or radio galaxy or an extended radio quasar has such a relatively large contribution from Fe $\Pi$ (e.g., see Osterbrock 1977). The second difference concerns the ratio of $[\mathrm{O} \mathrm{III}] \lambda 5007$ to $[\mathrm{O} \mathrm{II}] \lambda 3727$. There are indications in our data that this is significantly larger for the extended quasars than for the compact objects. Further work is in progress, and a more detailed discussion of this effect is deferred to a subsequent paper.

Differences between quasars having extended and compact radio emission could be related to a number of properties which differ between the two classes. Among the possibilities are the following: $(a)$ the presence of extended radio emission; (b) the different luminosities of the radio cores, which are typically two orders of magnitude less luminous in the extended radio sources; and $(c)$ the ratio of radio core to optical emission, which is also less in the extended systems. If either $(b)$ or $(c)$ was the main cause, one might expect the observed relations also to appear in the compact and extended objects treated separately. To pursue this, we plotted the line widths against both the radio core luminosities and the ratio of radio to optical emission in the core for each of the two groups; we did not see any effects in these relatively small samples. One method of narrowing the alternatives would be to compare our sample with a similar low-redshift sample of radio-quiet QSOs. These resemble extended sources in the sense that they have relatively weak core radio emission, though of course they do not have associated extended radio emission.

We can think of speculative explanations for the tendency for the widest and most irregular $\mathrm{H} \beta$ profiles to be preferentially associated with the extended radio sources. For example, since the extended sources could be older $\left(\geq 10^{8} \mathrm{yr}\right)$ than the compact ones $\left(\sim 10^{4} \mathrm{yr}\right)$, there could be larger energies involved in their production and maintenance. If the broad-line emitting region is closely connected with the primary energy source, the larger observed widths may be indicative of these larger energies. Also, during the $10^{8} \mathrm{yr}$ of activity, the extended source generation process may have carved out a region of relatively low pressure into which the broad-line emitting plasma can move more easily and faster. Another possibility is that the compact sources have considerable amounts of dust in their nuclei which prevent our seeing the broad-line emitting regions; older, extended sources could have dispersed or destroyed the dust.

We have considered objects having a relatively narrow range of optical luminosities $\left[P(\mathrm{H} \beta) \sim 10^{22}-10^{24}\right.$ $\left.\mathrm{W} \mathrm{m} \mathrm{m}^{-2} \mathrm{~Hz}^{-1}\right]$. Since the emission spectra are believed to be produced predominantly by photoionized gas, it is likely that the effects discussed here are intimately connected with the characteristics of the ionizing source. In the case of narrow-line radio galaxies, which generally do not have bright nuclei, there is presumably insufficient energy at their centers to power the broadline regions. It is important to make further analyses over a larger range of luminosities. Also, our sample is small and should be enlarged to provide a stronger basis for the relationships discussed here. Our object in this Letter has been to point out several suggestive correlations and to stimulate further work. A more systematic program to investigate these various effects is now under way at Lick.

We thank Howard French for help with the data reduction and Bill Burke and Don Osterbrock for useful discussions. G. K. M. acknowledges a travel grant from the Netherlands Organization for the Advancement of Pure Research (ZWO) and support from the Martin Kellogg Fund. This research was supported in part by NSF grant AST 76-20843.

\section{REFERENCES}

Bentley, M., Haves, P., Spencer, R. E., and Stannard, D. 1976, M.N.R.A.S., 176, 27.5 .

Burbidge, G. R., Crowne, A. H., and Smith, H. E. 1977, Ap. J. Suppl., 33, 113 .

Conway, R. G., Burn, B. J., and Vallee, J. P. 1977, Astr. Ap. Suppl., 27, 155.

Davis, R. J., Stannard, D., and Conway, R. G. 1977, Nature, 267, 596 .

Donaldson, W., Miley, G. K., Palmer, H. P., and Smith, H. 1969, M.N.R.A.S., 146, 213 .

Fanti, C., Fanti, R., Formiggini, L., Lari, C., and Padrielli, L. 1977, Astr. Ap. Suppl., 28, 351.

Knuth, D. E. 1969, in Seminumerical Algorithms (Menlo Park: Addison-Wesley), p. 41.
Lyne, A. G. 1972, M.N.R.A.S., 158, 431.

Miley, G. K. 1971, M.N.R.A.S., 152, 477 .

Miley, G. K., and Hartsuijker, A. 1978, Astr. Ap. Suppl., 34, 129.

Miller, J. S., Robinson, L. B., and Wampler, E. J. 1976, Adv. Electronics Electron Phys. (New York: Academic), 40B, 693.

Osterbrock, D. E. 1977, Ap.J., 215, 733.

Palmer, H. P., et al. 1967, Nature, 213, 789.

Phillips, M. M. 1978, Ap.J., 226, 736 .

Robinson, L. B., and Wampler, E. J. 1972, Pub. A.S.P., 84, 161.

Stannard, D., and Neal, D. S. 1977, M.N.R.A.S., 179, 71 .

Wardle, J. F. C., and Miley, G. K. 1974, Astr. Ap., 30, 305.

Wilkenson, P. N., Richards, P. J., and Bowden, T. N. 1974, M.N.R.A.S., 168,515 . 